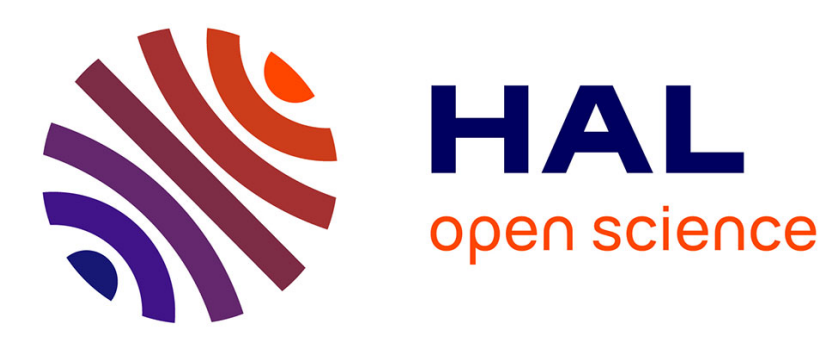

\title{
Optimal subharmonic emission of stable bubble oscillations in a tube
}

Yuzhe Fan, Haisen Li, Daniel Fuster

\section{To cite this version:}

Yuzhe Fan, Haisen Li, Daniel Fuster. Optimal subharmonic emission of stable bubble oscillations in a tube. Physical Review E , 2020, 102, 10.1103/PhysRevE.102.013105 . hal-02923744

\section{HAL Id: hal-02923744 \\ https://hal.science/hal-02923744}

Submitted on 27 Aug 2020

HAL is a multi-disciplinary open access archive for the deposit and dissemination of scientific research documents, whether they are published or not. The documents may come from teaching and research institutions in France or abroad, or from public or private research centers.
L'archive ouverte pluridisciplinaire HAL, est destinée au dépôt et à la diffusion de documents scientifiques de niveau recherche, publiés ou non, émanant des établissements d'enseignement et de recherche français ou étrangers, des laboratoires publics ou privés. 


\title{
Optimal subharmonic emission of stable bubble oscillations in a tube
}

\author{
Yuzhe Fan $\odot,{ }^{1,2,3,4}$ Haisen Li, ${ }^{1,2,3}$ and Daniel Fuster ${ }^{4, *}$ \\ ${ }^{1}$ College of Underwater Acoustic Engineering, Harbin Engineering University, Harbin 150001, China \\ ${ }^{2}$ Key Laboratory of Marine Information Acquisition and Security (Harbin Engineering University), Ministry of Industry and Information \\ Technology, Harbin 150001, China \\ ${ }^{3}$ Acoustic Science and Technology Laboratory, Harbin Engineering University, Harbin 150001, China \\ ${ }^{4}$ Sorbonne Université, UPMC Univ Paris 06, CNRS, UMR 7190, Institut Jean Le Rond d'Alembert, F-75005 Paris, France
}

(Received 27 March 2020; accepted 29 June 2020; published 14 July 2020)

\begin{abstract}
The subharmonic acoustic emission of a stable oscillating bubble inside a rigid tube is investigated by direct numerical simulation. The mechanisms of bubble-tube interaction on the acoustic wave emitted by the bubble are clarified. When the bubble is small compared to the tube diameter, a critical threshold for the pressure amplitude appears beyond the point which nonspherical effects become important and bubble breaks. For a finite tube diameter, the scattered wave by the bubble is shown to generate a plane wave where the intensity of the subharmonic component becomes maximum for an optimal distance between the bubble and the tube wall. This effect seems to be directly related to the appearance of local resonance phenomena and a bubble resonance shift where liquid's compressibility plays a major role.
\end{abstract}

DOI: 10.1103/PhysRevE.102.013105

\section{INTRODUCTION}

The subharmonic component of the acoustic wave scattered by a bubble is an important nonlinear mechanism in physical, chemical, and biological processes occurring in complex liquid environments $[1,2]$. The interest of detecting the subharmonic component of the signal is that, comparing to the tissue or other acoustic sources, the subharmonic can be almost uniquely created by bubbles, while the modification of the fundamental and superharmonic component of the signal can be modified by various different mechanisms. Thus, the use of subharmonics have been traditionally used to simplify the usually complex filtering procedures for imaging [3] and to easily classify the bubble cluster response during sonoluminescence [4-6].

In classical models used to discuss the appearance of a subharmonic the bubble is assumed to be spherical, so that the bubble radius temporal evolution $R(t)$ can be described using a Rayleigh-Plesset-like (RP-like) equation [7]:

$$
\rho_{l_{0}} R \ddot{R}+\frac{3}{2} \rho_{l_{0}} \dot{R}^{2}=p_{b}-p_{e}-\rho_{l_{0}} \frac{\partial \phi_{\text {int }}}{\partial t},
$$

where $\rho_{l_{0}}$ is the external liquid density which is assumed to be constant, $p_{b}$ is the pressure inside the bubble, $p_{e}$ is the external pressure in the liquid phase, and $\phi_{\text {int }}(t)$ is the interacting potential.

The limiting case of $\phi_{\text {int }}(t)=0$ (e.g., a spherical bubble oscillating in an infinite amount of liquid) the subharmonic emission by a bubble has been widely investigated. For instance initial studies were sought to understand the threshold of the appearance of subharmonics [8-10], the influence of the

\footnotetext{
*fuster@dalembert.upmc.fr
}

ambient pressure on subharmonic emission [11-13], and the optimal driving waveform [14-16].

However, in many natural environments and industrial applications, the bubble oscillates interacting with other bubbles or the presence of solid walls that prevents neglecting the interaction term $\phi_{\mathrm{int}}(t)$ [17]. This interaction term can significantly influence the predictions of the frequency spectrum of the acoustic wave emitted by bubbles [18-20], revealing the importance of modeling this term correctly. Jiang et al. [21] show that the interaction between two bubbles imposes an extra nonlinear influence on bubble oscillation, which can be observed by a development of the subharmonic component on the frequency spectra of the acoustic emission signal. Guédra et al. [22] has extended Prosperetti analyses to a bubble inside a bubble cluster to obtain an analytical expression of the subharmonic threshold accounting for direct bubble-bubble interactions. The main conclusion is that the presence of surrounding bubbles induce a shift on the subharmonic resonance frequency and decrease of the pressure threshold required to observe the appearance of subharmonics.

These studies rely on the correct representation of $\phi_{\text {int }}$ by a simplified model, which is not always straightforward. Supposing that (i) the bubble radius is small in comparison with the wavelength of the external driving pressure and (ii) the averaged distance between the bubbles is small compared to the wavelength, the interaction term is usually calculated taking advantage of the incompressible assumption in the bubble near field [23-26]. This approach is problematic when the number of bubbles is large because the interaction term diverges $[27,28]$ and one needs to introduce a cutoff distance within which bubble-bubble interactions effectively occur [29]. An alternative physical approach to resolve the paradox is to account for liquid compressibility [30]. In the context of bubble screens Leroy et al. [31] propose a solution for the linearized problem. Alternatively, Miksis and Ting [32] 
propose to obtain $\phi_{\text {int }}(t)$ from leading order terms related to liquid compressibility using a multiscale approach still using a locally incompressible assumption in the near field. Whether the incompressible or compressible interaction mechanism prevails in a general configuration is not fully clarified. In addition, a common problem of all RP-like models is that in the nonlinear regime the bubble is expected to oscillate nonspherically due to the presence of the tube.

In this study, we use direct numerical simulations (DNS) to clarify the capability of simplified models to capture the acoustic emission of subharmonics by bubble oscillations in a tube. The bubble oscillation amplitude and the frequency spectra of the pressure wave emitted by the bubble are presented as a function of the most relevant dimensionless parameters: the forcing amplitude, the Weber number, and the ratio between the bubble radius and the tube diameter.

\section{PHYSICAL MODELS}

\section{A. Full model}

In this section, a brief description of the DNS method used is given. The reader is referred to Ref. [33] for further details. The method is implemented in the open source solver Basilisk [34] and it has yielded excellent agreement with experimental results. We solve for the Navier-Stokes equations for a mixture of two immiscible substances where the position is defined by a Heaviside function $\mathcal{H}$ that takes the value of 1 in the reference phase. The interface position is then given by the solution of an advection equation for $\mathcal{H}$

$$
\frac{\partial \mathcal{H}}{\partial t}+\mathbf{u} \cdot \nabla \mathcal{H}=0 \text {. }
$$

The model is based on the six equation model that solves for the continuity and energy equations for the $i$ th phase ( $i \in g, l$ corresponding to the gas phase and the liquid phase) and the averaged momentum equation. In this paper we restrict ourselves to situations where thermal diffusion and mass transfer effects are not relevant so that the final system becomes

$$
\begin{gathered}
\frac{\partial \rho_{i}}{\partial t}+\nabla \cdot\left(\rho_{i} \mathbf{u}_{i}\right)=0, \\
\frac{\partial \rho \mathbf{u}}{\partial t}+\nabla \cdot(\rho \mathbf{u u})=-\nabla p+\nabla \cdot \tau_{i}^{\prime}+\sigma \kappa \nabla c, \\
\frac{\partial \rho_{i} e_{i}+1 / 2 \rho_{i} \mathbf{u}_{i}^{2}}{\partial t}+\nabla \cdot\left(\rho_{i} e_{i} \mathbf{u}_{i}+1 / 2 \rho_{i} \mathbf{u}_{i}^{2}\right) \\
=-\nabla \cdot\left(\mathbf{u}_{i} p_{i}\right)+\nabla \cdot\left(\tau_{i}^{\prime} \mathbf{u}_{i}\right),
\end{gathered}
$$

where $\mathbf{u}_{i}$ is the fluid velocity vector in each phase, $\tau_{i}^{\prime}$ is the viscous stress tensor depending on the dynamic viscosity $\mu_{i}$, $e$ denotes the internal energy, and $\kappa$ is the curvature of the interface.

The system of equations solved is closed by adding an equation of state (EOS) for each phase that establishes the relation between the various thermodynamic variables $\operatorname{EOS}_{i}\left(p_{i}, T_{i}, \rho_{i}\right)=0$ in the Mie-Gruneisen form

$$
\rho_{i} e_{i}=\frac{p_{i}+\Gamma_{i} \Pi_{i}}{\Gamma_{i}-1},
$$

where $\Gamma_{i}$ and $\Pi_{i}$ are empirical constants.

\section{B. RP-like models}

The model above can be further reduced if we assume that the bubble is spherical and we consider the liquid as a slightly compressible substance. For a bubbly freely oscillating, the radius temporal evolution can be described by the KellerMiksis (KM) equation [35]. When tube wall exists, it is required to introduce an additional interaction term capturing for the influence of the wall on the pressure effectively felt by the bubble. The resulting ( $\mathrm{KM}+\mathrm{Int})$ equation is as follows:

$$
\begin{gathered}
\rho_{l_{0}} R \ddot{R}\left(1-\frac{\dot{R}}{c_{l_{0}}}\right)+\rho_{l_{0}} \frac{3 \dot{R}^{2}}{2}\left(1-\frac{\dot{R}}{3 c_{l_{0}}}\right) \\
=\left(1+\frac{\dot{R}}{c_{l_{0}}}+\frac{R}{c_{l_{0}}} \frac{d}{d t}\right)\left(p_{b}-p_{e}\right)-\rho_{l_{0}} \frac{\partial \phi_{\text {int }}}{\partial t}, \\
p_{b}=\left(p_{l_{0}}+\frac{2 \sigma}{R_{0}}\right)\left(\frac{R_{0}}{R}\right)^{3 \gamma}-\frac{2 \sigma}{R}-\frac{4 \mu_{l}}{R} \dot{R}-p_{l_{0}},
\end{gathered}
$$

where $c_{l_{0}}$ is the speed of sound in the liquid which is assumed to be constant and $p_{l_{0}}$ is the static pressure.

In the vecinity of a freely oscillating bubble the pressure emitted by the bubble at a distance $r$ from the center bubble $p_{\text {rad }}$ can be obtained from the Euler equations in the liquid under the incompressible assumption:

$$
p_{\text {rad }}(r)=\rho_{l_{0}} \frac{1}{r} \frac{d}{d t}\left(R^{2} \dot{R}\right)+\mathcal{O}\left(\frac{1}{R^{4}}\right) .
$$

If the tube wall is relatively close and the pressure generated by the presence of the walls is estimated using Eq. (9), then the interaction term is

$$
\frac{\partial \phi_{\text {int }}}{\partial t}=\frac{C_{1}}{D}\left(2 \dot{R}^{2} R+R^{2} \ddot{R}\right)
$$

where $C_{1}$ is a constant to be determined and $D$ is the tube diameter. Taking $C_{1}=2 \pi$, this model is similar to the one used by Mettin et al. [23] to model the incompressible mechanism of bubble-bubble interactions in bubble screens. In the following this model, which neglects liquid compressibility effects in the interaction term, will be used as reference to discuss the results obtained from DNS.

\section{NUMERICAL RESULTS}

\section{A. Problem description}

In this work we simulate numerically the dynamics of a single bubble oscillation inside a tube (Fig. 1). We consider an axisymmetric system with a rigid reflecting boundary at a distance $D / 2$ from the bubble center and periodic boundaries along the $x$ direction. A sine pulse with a Gaussian-shaped envelope is used as the incident wave, so that the pressure pulse at the bubble location $(x=0)$ becomes

$$
\frac{p_{e}(t)}{p_{l_{0}}}=1-P_{a}\left(\sin (\omega t) \exp \left[-\frac{\left(t-t_{0}\right)^{2}}{2 b t_{0}^{2}}\right],\right.
$$

where $P_{a}$ is the amplitude of the incident pulse, $t_{0}=N_{\text {cycles }} \frac{\lambda}{2 c_{l_{0}}}$ ( $N_{\text {cycles }}$ is the number of the incident pulse cycles and $\lambda$ is the wavelength of the pulse) and $b=1 / 3$. A $N_{\text {cycles }}=6$ cycles driving pulse is chosen in order to limit the use of computational resources. 


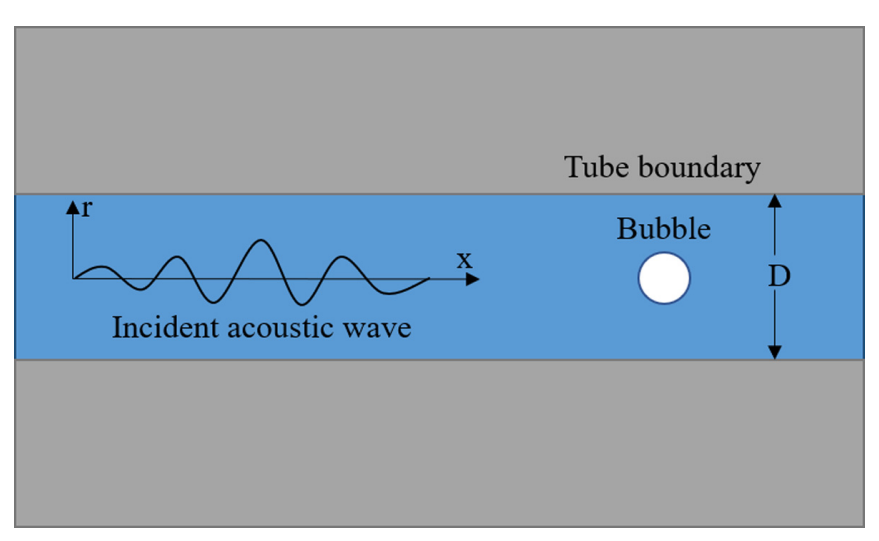

FIG. 1. Illustrating bubble dynamics inside a tube.

The problem is rendered dimensionless using the bubble radius, the liquid density and ambient pressure as the characteristic values of the problem. Thus, it is possible to express the solution of the dimensionless radius as a function of the following dimensionless parameters:

$$
\frac{R}{R_{0}}=f\left(P_{a}, \frac{\omega}{\omega_{N}}, K, \text { We, Re, } \frac{D}{R_{0}}\right),
$$

where we characterize the forcing wave by the ratio between characteristic forcing frequency $\omega$ and the natural bubble resonance frequency $\omega_{N}=\frac{1}{R_{0}} \sqrt{3 \gamma \frac{p_{0}}{\rho_{l_{0}}}+2(3 \gamma-1) \frac{\sigma}{\rho_{l_{0}} R_{0}}}$. The rest of dimensionless quantities correspond to the Weber number, We $=p_{l_{0}} R_{0} / \sigma$, the Reynolds number, $\operatorname{Re}=\rho_{l_{0}} U_{0} R_{0} / \mu_{l}$ where $U_{0}=\sqrt{p_{l_{0}} / \rho_{l_{0}}}$, and the ratio of bulk compressibilities $K=\frac{\rho_{g_{0}} c_{g_{0}}^{2}}{\rho_{l_{0}} c_{l_{0}}^{2}}$. For the sake of simplicity, we set a constant Reynolds number for all simulations $\mathrm{Re}=50$ and we chose an incident pressure wave with $\frac{\omega}{\omega_{N}}=2$. Additional calculations not shown in this manuscript show that changes in the Reynolds number do not modify the conclusions reached in this manuscript.

An ideal gas is considered $\left(\Pi_{g}=0\right.$ and $\left.\Gamma_{g}=\gamma=1.4\right)$ while the parameters of the EOS in the liquid are $\Gamma_{l}=7.14$ and $\Pi_{l} / p_{l_{0}}=\frac{\Gamma_{g}}{\Gamma_{l} K}-1$. The value of $K$ will be $K=6.5 \times$ $10^{-4}$ in most of the simulations included in this manuscript except specified otherwise. Note that this value is larger than that of air-water systems $\left(K_{a / w}=4 \times 10^{-5}\right)$ in order to limit the wavelength of the incident pulse and therefore reduce the computational time. Under these conditions, the solution only depends on three dimensionless numbers: We, $P_{a}$, and $D / R_{0}$.

For DNS calculations the domain size $L_{d}$ in the $x$ direction is $L_{d} / R_{0} \approx 450$. An static and nonuniform grid is used where the minimum grid size is set to $\Delta / R_{0} \approx 1 / 36$. The solution is shown not to depend to the grid size for smaller grid sizes.

\section{B. A freely single oscillating bubble}

We start considering a case where the bubble is far from the wall $\left(D / R_{0} \approx 900\right)$ to avoid any spurious effects from the reflection in the boundaries $(D / 2 \lambda>3)$. This case is close to the limit of a single bubble oscillation in an unbounded domain where the capability of KM models to predict the appearance of subharmonics has been already verified by DNS [36].

The bubble response is characterized by an effective bubble radius, defined as the radius of a spherical bubble with volume $V_{\text {bub }}$ obtained from DNS at instant $t, R_{e}(t)=\left(\frac{3 V_{\text {bub }}}{4 \pi}\right)^{1 / 3}$. The pressure wave scattered by the bubble is obtained from DNS in the axis of symmetry at a distance $x_{s} / R_{0}=15$ from the bubble center. The Fourier transformation is applied to the pressure difference between the pressure obtained in the presence and in absence of a bubble to obtain the emitted pressure in the frequency domain $\tilde{p}$. From this value we obtain the energy in the frequency domain (in dB) as $E=20 \log _{10}\left(\frac{\tilde{p}}{\tilde{p}_{\max }}\right)$, where $E_{0}=20 \log _{10}\left(\tilde{p}_{\max }\right)=2.5 \mathrm{~dB}$ corresponds to the maximum energy in the fundamental component obtained among all simulations in the manuscript. The pressure predicted by the $\mathrm{KM}$ model is obtained using the radiated pressure at a distance $x_{s}$ from the bubble [Eq. (9)].

Figures 2 and 3 show the interface contour and the time evolution of the effective bubble radius $R_{e}(t)$ for $P_{a}=6$ and $\mathrm{We}=7$. The difference between the effective radius predicted by the DNS and KM model increases as time progresses. Note that, due to the Gaussian-shaped envelope used, the amplitude of the incident wave increases before the bubble volume reaches its minimum value (around $t=4.5$ ), and decreases after that. The bubble shape is visually spherical during the growth phase, and develops nonspherical modes during the compression phase due to Rayleigh-Taylor instabilities. Nonspherical effects gradually influence the bubble oscillation even in the growth stage due to parametric instabilities [37], this being the main reason making the results calculated by the DNS and the KM equation differ.

Figure 4 compares the energy spectrum of the radiated wave calculated by DNS and predicted from the KM equation. The fundamental harmonic is relatively well captured by the KM model, while it tends to overpredict the subharmonic amplitude. To gain further insight about the influence of subharmonic we perform a parametric study on the influence of the incident amplitude $P_{a}$ and the Weber number (Fig. 5). Three characteristic regimes are observed: at low forcing amplitudes (but above the subharmonic appearance threshold) the subharmonic is visible but its amplitude remains relatively small irrespective of the forcing amplitude. Then, the amplitude of the subharmonic grows fast and finally saturates for large forcing amplitudes. These observations are consistent
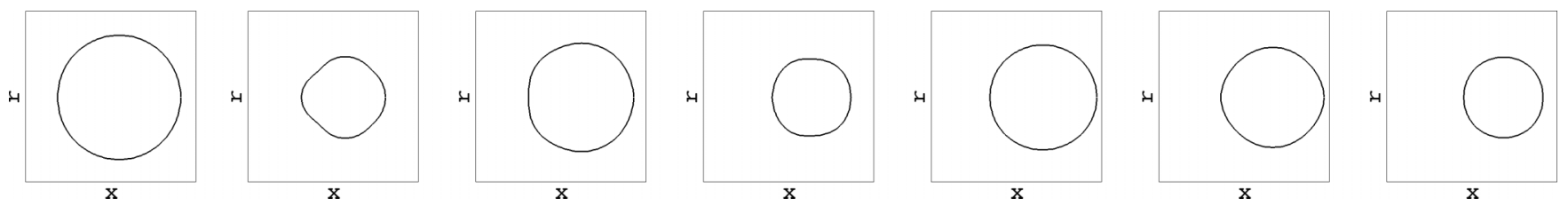

FIG. 2. Interface contours for $P_{a}=6$ and $\mathrm{We}=7$ at $t=4,4.8,5.4,6.2,6.8,7.6$ The box length in all images is $\frac{L_{\mathrm{box}}}{R_{0}}=4$. 


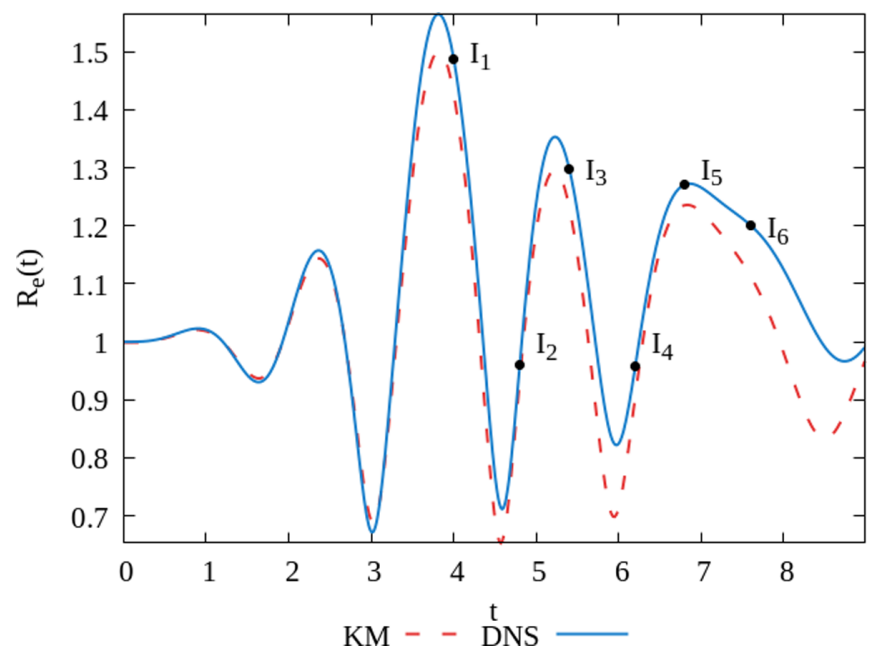

FIG. 3. Comparison of the effective bubble radius $R_{e}(t)$ between DNS and KM equation for $P_{a}=6$ and $\mathrm{We}=7$. The points labeled as $I_{n}$ with $n \in 1,2, \ldots, 6$ correspond to the interface isocontours shown in Fig. 2.

with experimental observations [38]. The predictions of DNS and the KM model are close to each other for sufficiently small amplitudes, KM tending to underpredict the amplitude of the subharmonic. Indeed the differences between KM and DNS results increase by increasing the forcing amplitude. This effect is mainly attributed to the development of nonspherical modes mentioned above. These perturbations are eventually responsible of the bubble fragmentation limiting the maximum amplitude for which a stable subharmonic emission is observed for a given We number. The amplitude threshold below which stable oscillations occur, shown with a dashed line, strongly depends on the Weber number and it is also expected to be dependent on the number of cycles used. The Weber number also influences the intensity of the subharmonic amplitude. This is because the parameter controlling the strength of the excitation has to be corrected to account for the pressure excess of the Laplace term $[8,9]$.

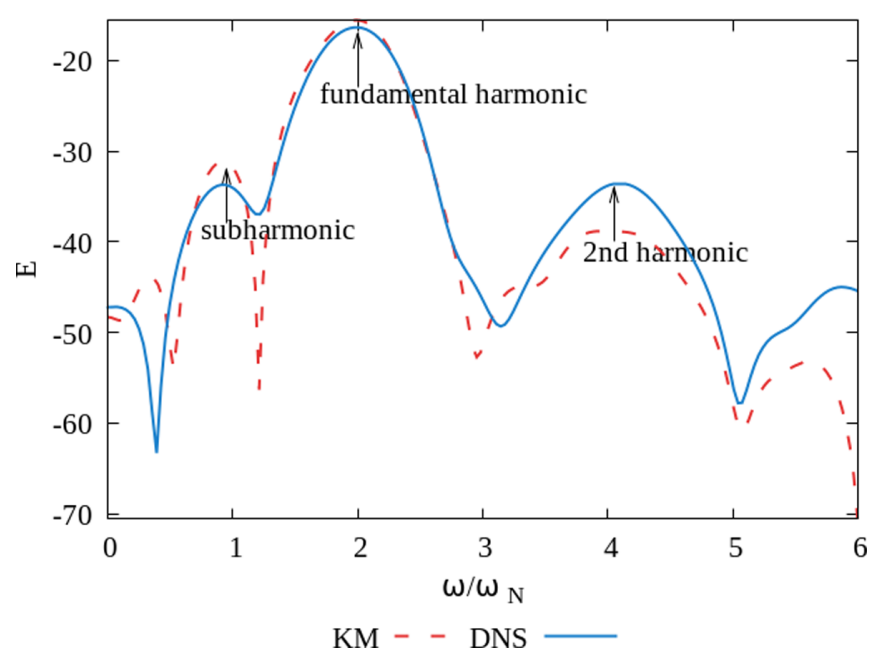

FIG. 4. Frequency spectrum of the pressure wave emitted by the bubble at $P_{a}=6$ and $\mathrm{We}=7$.

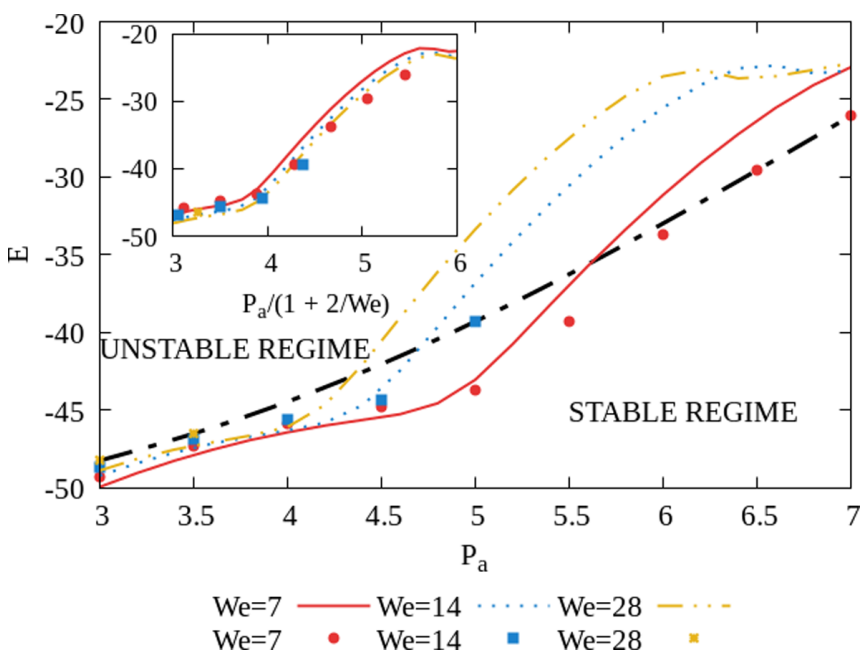

FIG. 5. Energy in the subharmonic component as a function of the forcing amplitude for various We. Results obtained from DNS (dots) and the KM equation (lines). The stable regime and the unstable regime are separated by the black dash-dot line. In the inset we show the same plot but using a rescaled amplitude in the $x$ axis.

As shown in the inset of Fig. 5 we see that using the corrected amplitude $P_{a} /(1+2 / \mathrm{We})$ all curves collapse irrespective of the value of We number. Thus, for a system with given physical properties, the larger the We the stronger the subharmonic for a constant $P_{a}$ (e.g., large bubbles are more prompt to generate subhamonics) but large values of We can promote the appearance of nonspherical modes that eventually break the bubble into small fragments.

\section{Wall effects}

We focus now on the influence of the presence of the tube wall on the dynamic response of the bubble and subharmonic emission.

The influence of the interactions can be discussed in terms of the dimensionless distances $D / R_{0}$ and $k D$, where $k$ is the wave number. Note that for the particular case of $\omega=$ $2 \omega_{N}$, both parameters are related through the ratio of bulk compressibilities as

$$
k D=\frac{D}{R_{0}} \frac{2 R_{0} \omega_{N}}{c_{l_{0}}} \approx \frac{2 D}{R_{0}} \sqrt{3 K}=\widetilde{k D}
$$

Most of of the models accounting for interactions assume that $\widetilde{k D} \ll 1$ removing the explicit dependence of this parameter and leaving $D / R_{0}$ as the relevant parameter influencing the bubble response. This is indeed the case of the KM + Int model considered here which assumes the liquid in the near field as incompressible.

Figure 6 shows the temporal evolution of the bubble radius for $D / R_{0} \approx 19, K=6.5 \times 10^{-4}(\widetilde{k D} \approx 1.68), P_{a}=4$, and $\mathrm{We}=7$. We observe that the amplitude of the oscillation is indeed larger than the results from DNS in an unbounded domain, indicating the appearance of local resonance established between the bubble and the tube wall similar to the one reported in previous works of bubbles in closed pores [39].

A finer analysis on the optimal value of $\widetilde{k D}$ is shown in Fig. 7 where we vary this parameter by changing the distance 


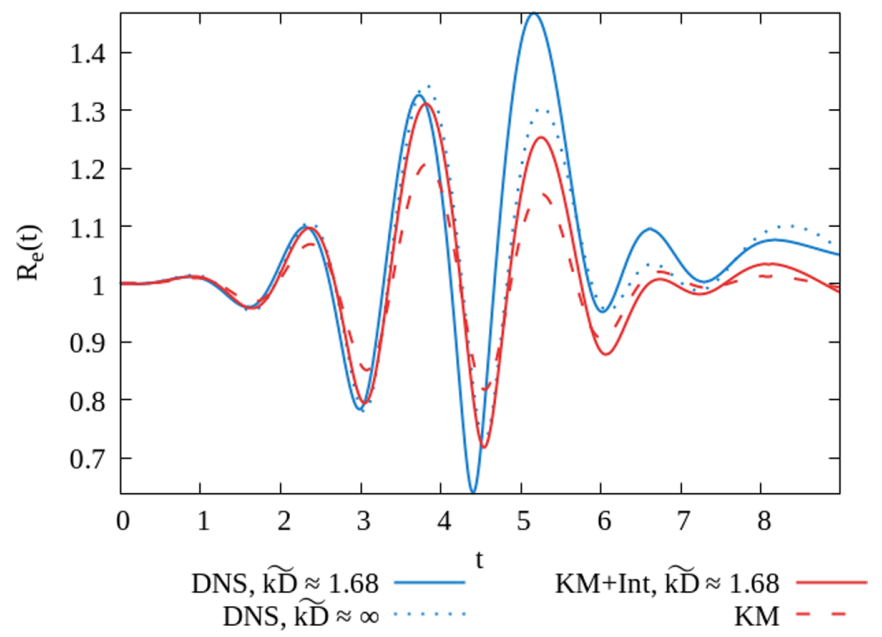

FIG. 6. Comparison of the effective bubble radius evolution for $P_{a}=4$, We $=7$ in a tube $\left(D / R_{0}=19\right)$ and in a free field. The predictions of the $\mathrm{KM}$ equation with and without interaction term are included for reference.

$D / R_{0}$ for a constant value of $K=6.5 \times 10^{-4}$. The amplitude of the oscillation displays a maximum for a relatively small distance, $D / R_{0} \approx 19$, that corresponds to $\widetilde{k D}_{\mathrm{opt}} \approx 1.68$. From Fig. 7 we can indeed see that the influence of $D / R_{0}$ on the amplitude of the bubble oscillation is not well captured by the $\mathrm{KM}+$ Int model, which predicts a decrease on the amplitude of the oscillations when $D / R_{0}$ decreases due to confinement effects. This effect is not surprising as the KM model with interactions neglects any influence of phase lag on the results. Figure 8 shows that the optimal distance shifts to lower values as $K$ increases revealing that the local resonance conditions found do not correspond to a universal value of $\widetilde{k D}\left(\widetilde{k D}_{\mathrm{opt}} \approx 2.49\right.$ when $K \approx 32.6 \times 10^{-4}$ and $\widetilde{k D}_{\mathrm{opt}} \approx 1.68$ when $\left.K \approx 6.5 \times 10^{-4}\right)$. This implies that the mechanism of

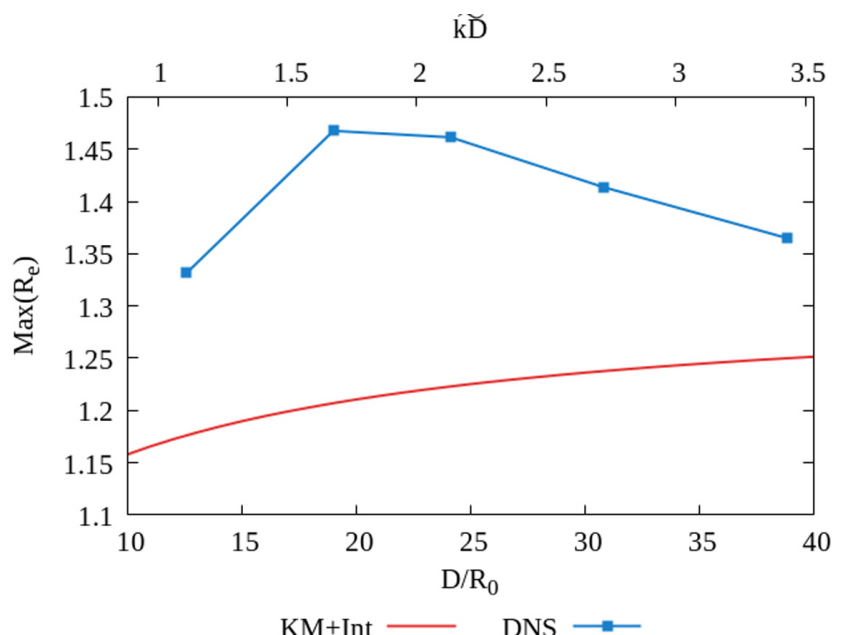

FIG. 7. Maximum amplitude of the bubble radius oscillation as a function of the distance between the bubble and the tube wall (bottom axis) and $\widetilde{k D}$ (top axis) obtained by DNS and the KM model in the stable oscillation regime (no fragmentation). $P_{a}=4$, We $=7, K=$ $6.5 \times 10^{-4}$.

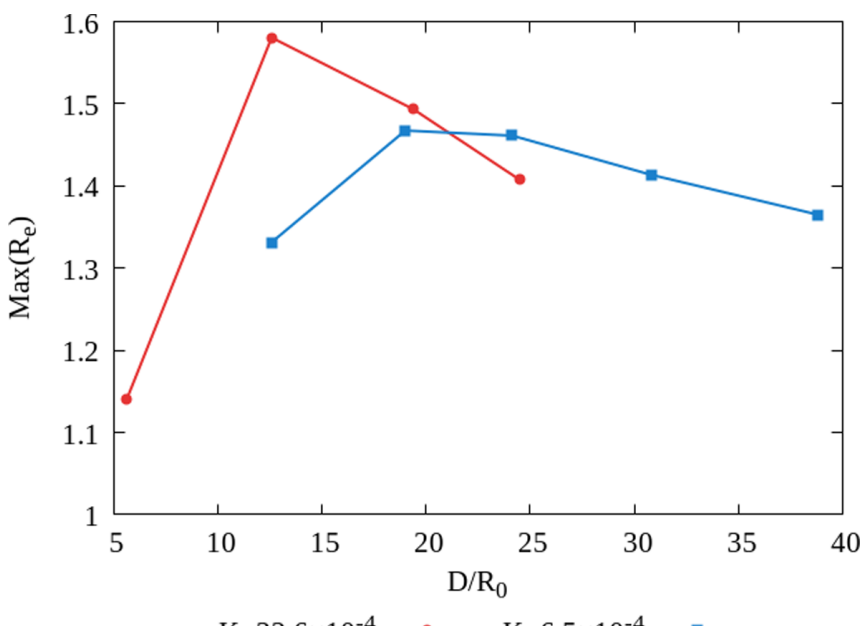

FIG. 8. Influence of $K$ on the optimal condition of the maximum effective bubble radius evolution for $P_{a}=4, \mathrm{We}=7$.

interaction cannot be discussed in terms of resonance effects associated to $k D$ only. This effect is attributed to a shift in the resonance frequency in the presence of the wall, $\omega_{N \text {,int }}$, which eventually modifies the amplitude of the bubble oscillation (see Appendix B). The frequency shift can depend on both, $k D$ and $D / R_{0}$. Previously published works pointing out in this direction include investigations of the amplitude of the linear bubble oscillation in bubble screens [31]: While a shift for lower frequencies is expected from in-phase oscillations when the bubbles are close to each other

$$
\omega_{N, \text { int }}=\frac{\omega_{N}}{\sqrt{1+\frac{R_{0}}{D}[\cos (k D)]}},
$$

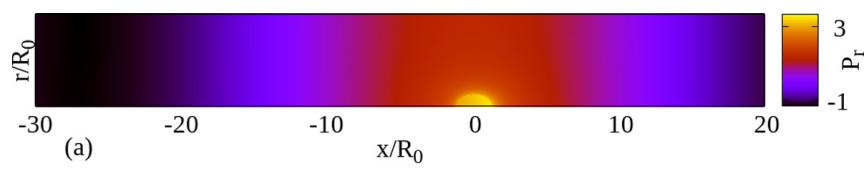

(a)

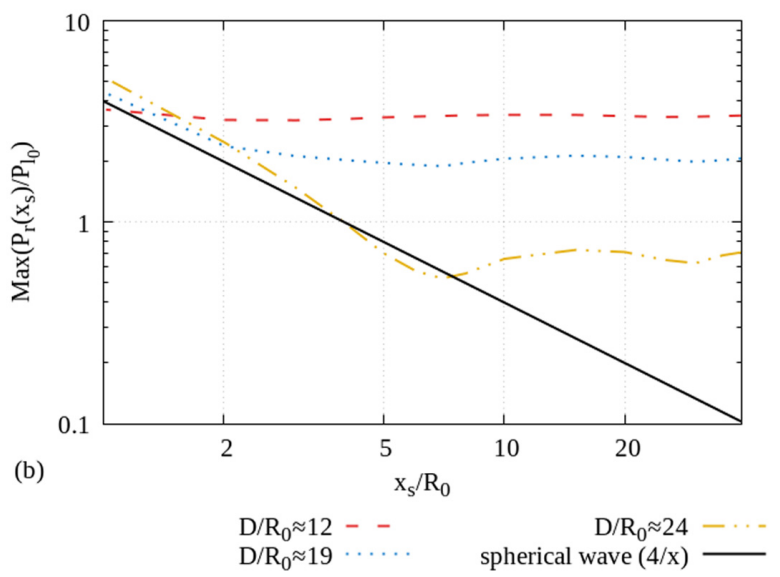

FIG. 9. Top: Pressure field induced by the bubble, $p_{r}=p_{l}-p_{\infty}$, in the $x$ - $r$ domain $\left(P_{a}=4 \mathrm{We}=7, D / R_{0} \approx 19\right)$. Bottom: Influence of the bubble-wall distance on the maximum amplitude of the reflected pressure wave as a function of the distance to the bubble center $\left(P_{a}=4\right.$, We $\left.=7\right)$. 

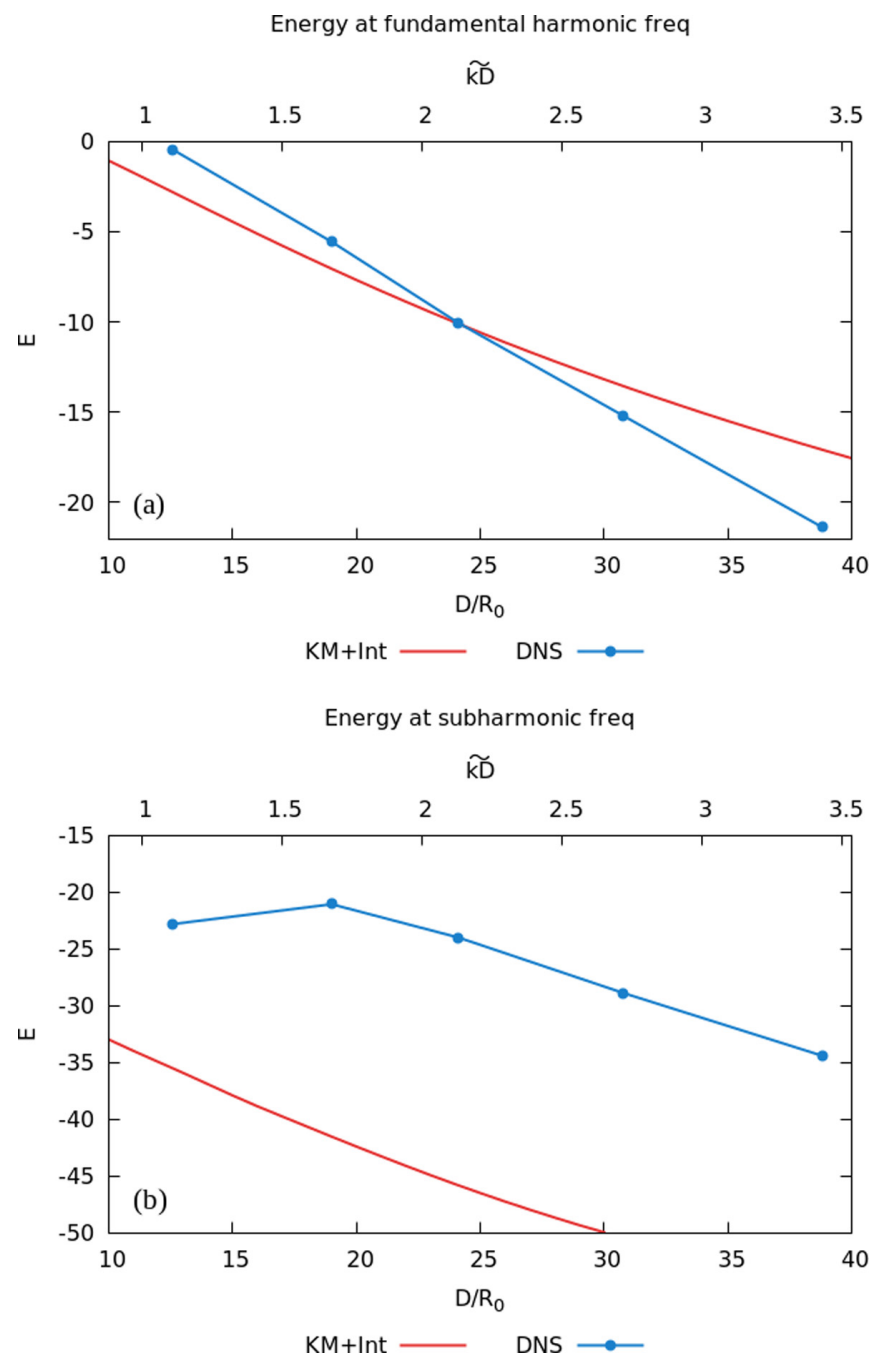

FIG. 10. Influence of the distance between the bubble and the tube wall in the energy in the fundamental component (top) and subharmonic (bottom). $P_{a}=4, \mathrm{We}=7$.

a net shift toward higher frequencies can be experimentally observed when phase and antiphase interaction occur [31],

$$
\omega_{N, \text { int }}=\frac{\omega_{N}}{\sqrt{1-2 \sqrt{\pi} \frac{R_{0}}{D}}} .
$$

These result provide some evidences about why the results shown in Fig. 8 depend on both $k D$, which certainly modify the pressure field due to local resonance conditions, and $D / R_{0}$, which modifies the bubble resonance frequency. We recall that these results are not directly applicable to our problem here as these equations are found for similar but still different physical problems. In addition, the situation considered in this manuscript is more involved as nonlinear effects are relevant although it is important to mention that additional tests show that optimal conditions also appear in the linear regime.

Once the influence of the wall on the bubble response has been described, and before investigating the subharmonic emission, it is also important to characterize the structure of the pressure wave emitted by the bubble. Figure 9 shows the pressure field emitted by the bubble at a given instant and

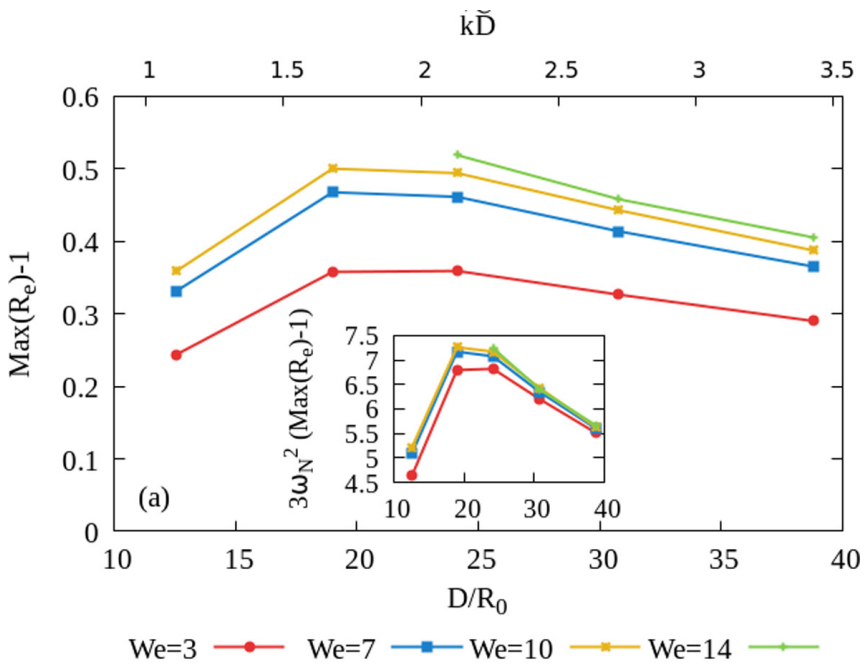

$\mathrm{k} \breve{\mathrm{D}}$

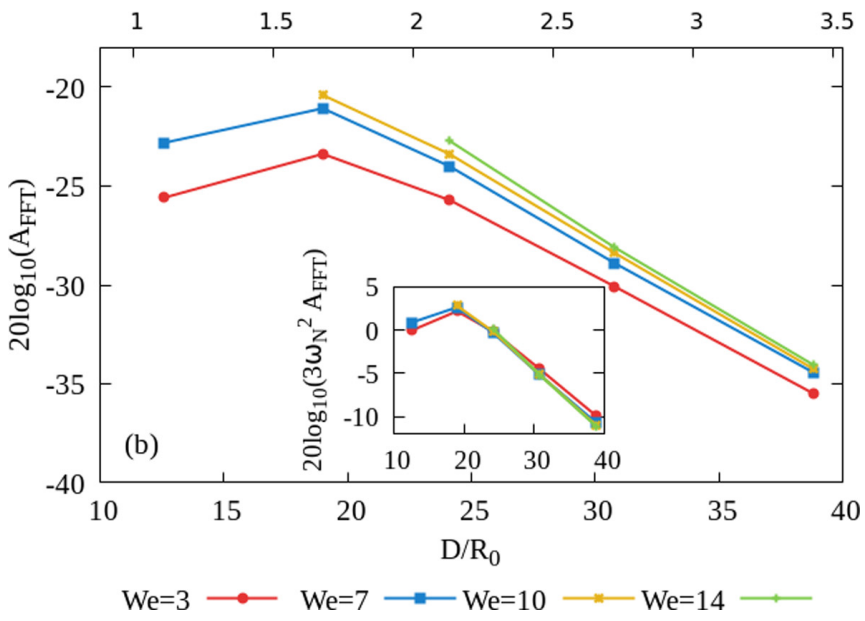

FIG. 11. Maximum $R_{e}(t)$ (top) and intensity of the subharmonic (bottom) as a function of $D / R_{0}$ and We for $P_{a}=4$. For reference the values of $\widetilde{k D}$ are shown in the upper axis.

the transition from the spherical source to the plane wave by sampling the reflected pressure in the symmetry axis at differences distances $x_{s} / R_{0}$. We distinguish two clear regions: the near field region where the pressure corresponds to an spherical outgoing wave and the pressure decreases with the inverse of the distance, and the far field where the interference phenomena caused by wave reflection at the tube wall quickly lead to the appearance of a planar wave where the amplitude stays constant as it propagates along the tube. From the results in Fig. 9(b) we can conclude that by increasing $D / R_{0}$, (i) the transition distance from a spherical to a planar wave increases and (ii) the amplitude of the reflected wave decreases roughly as $R_{0} / D$, which is the result of the reflection of bubble screens to linear pressure pulses [40]. In the following, to obtain a stable plane wave, the pressure is sampled at distance $\left|x_{s}\right| / R_{0}=15$ in DNS and in order to compare the results with the predictions of the $\mathrm{KM}+$ Int model, we use a simple semiheuristic model described in Appendix A to relate the bubble response obtained from KM + Int equation with the pressure amplitude of the plane wave emitted by the bubble. 

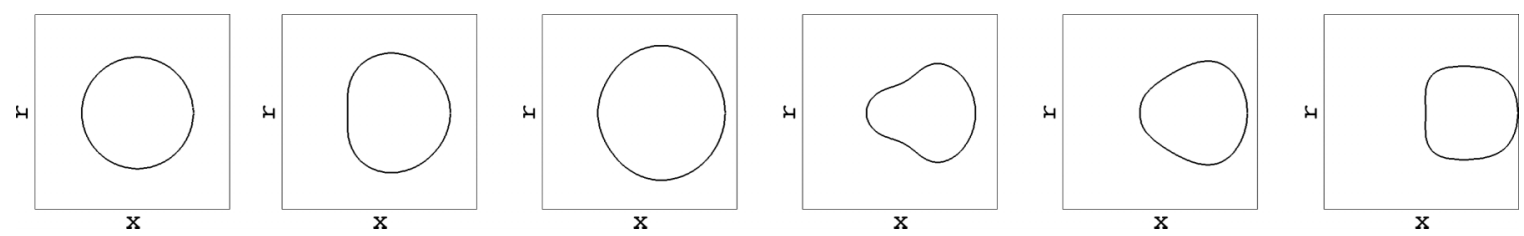

FIG. 12. Interface contours of the bubble at different instants for $P_{a}=4, \mathrm{We}=7$, and $D / R_{0}=19$. The frametimes (from left to right) are $t=4,4.8,5.4,6.2,6.8,7.6$ (Both $x$-axis and $r$-axis range from -2 to 2 ).

Figure 10 shows the influence of the nondimensional distance $D / R_{0}$ on the energy in the fundamental and subharmonic component for $P_{a}=4, \mathrm{We}=7$, and $K=6.5 \times 10^{-4}$. DNS results show that for the resonance distance found previously $D / R_{0} \approx 19$, the subharmonic amplitude is also maximum; for larger distances, the plane wave generated by the bubble is less intense mainly because for large values of the $D / R_{0}$ the relative importance of the bubble on the overall transmission and reflection decreases. Comparing the results of DNS with the predictions of the simplified KM + Int models accounting for bubble-wall interactions, we can see that the fundamental is indeed well captured both qualitatively and quantitatively for small-enough distances, when the structure of the plane wave is well established. At large distances the model presented in Appendix A used to predict the structure of the plane wave is not applicable and a procedure similar to the one followed previously for a free oscillating bubble should be followed. The predictions of the KM + Int model fails capturing the amplitude of the subharmonic component as a consequence of the resonant behavior discussed above. Generally speaking, the influence of interactions is observed to play a major role on the correct prediction of the appearance and intensity of subharmonics compared to the impact on the fundamental harmonic.

Like in the case of a bubble in a free field discussed in the previous section, We plays a role on the amplitude of the subharmonic and the maximum effective bubble radius (Fig. 11). For a constant value of $P_{a}$, the amplitude of the amplitude of the oscillation and the intensity of the subharmonic increases as Weber increases until the development of nonspherical

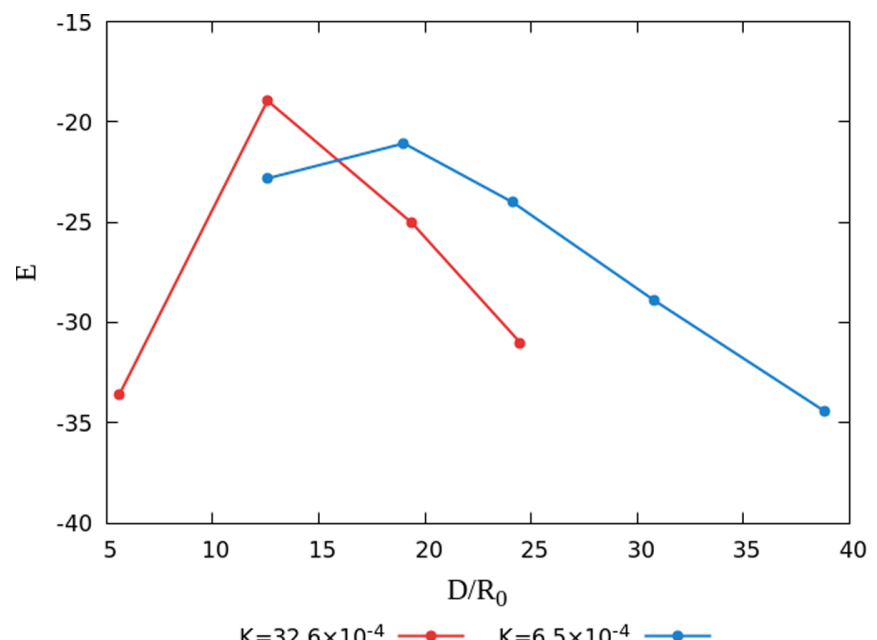

FIG. 13. Influence of $K$ on the maximum Intensity of the subharmonic. We $=7$ for $P_{a}=4$. modes fragment the bubble. As shown in the inset of Fig. 11 we see that it is possible to rescale the amplitude using an scaling factor that in dimensionless form becomes $3 \omega_{N}$ (see Appendix B for derivation and dimensional form). Using this factor it is possible to collapse all curves irrespective of the value of We number. By comparing the interface isocontours of Figs. 12 and 2 we see that the presence of the wall clearly enhances the appearance of nonspherical modes. For We $<10$ the optimal distance for which the intensity of the subharmonic is maximal $\left(D / R_{0} \approx 19\right)$ is found to be not dependent on We. For We $>10$ such distance cannot be reached in a stable configuration and the bubble breaks. We therefore conclude that for a fixed forcing amplitude, the optimal conditions to measure the appearance of subharmonics correspond to We $=10$ and $D / R_{0} \approx 19$. That being said, we note that we cannot assess where the overall maximum among all values of $P_{a}$ is found, as small bubbles (small We) may be stable at higher amplitudes $P_{a}$.

Finally, we recall that the value of $K$ influences the optimal condition. Thus, we find that the optimal distance reported in Fig. 13 is shifted to a smaller value than that which would be obtained for air-water systems. This result also supports that the optimal condition is a function of both characteristic distances $k D$ and $D / R_{0}$.

\section{CONCLUSIONS}

A DNS study has been carried out to investigate the sensitivity of bubble acoustic emission to bubble-wall interactions and nonspherical deformations. Simulations show that when the bubble is in a free field configuration the predictions of both DNS and KM models are close to each other for forcing amplitudes $P_{a}$ below a threshold beyond which the development of nonspherical modes eventually break the bubble.

For a finite tube diameter, the scattered wave by the bubble reflects back into the tube walls influencing the response of the bubble itself. Local resonant phenomena has been shown to play a major role on determining the amplitude of the bubble oscillation but a shift in the resonance frequency related to both, $k D$ and $D / R_{0}$, also influence the amplitude of the oscillations. We find that an optimal distance $D / R_{0}$ exists where the amplitude of the bubble oscillation can be even larger than that of a bubble in a free field configuration and where the subharmonic amplitude is maximal. For distances larger than this optimal distance, the wave scattered by the tube becomes weaker and the interaction term become less important, so does the bubble acoustic emission. For smaller distances the presence of the wall exerts a strong effect that eventually leads to a decrease on the oscillation amplitude and a decrease of the subharmonic amplitude. Based on a further 
parameter test, this optimal always appears irrespective of the values of We, although its value is important to determine whether the bubble breaks or not. The results also show the difficulties to reproduce DNS results of the classical model to account for the interaction between the bubble and the tube assuming liquid as an incompressible substance.

\section{ACKNOWLEDGMENTS}

D.F. acknowledge the fruitful discussions with Agnes Maurel, Kim Pham, and Jean François Mercier about the influence of compressibility effects on the dynamics of bubbles in bubble screens. This work has been supported by Project No. 41576102 by the NSFC and Project No. 3072019GIP0506 supported by Ph.D. Student Research and Innovation Fund of the Fundamental Research Funds for the Central Universities.

\section{APPENDIX A: PLANE-WAVE EMISSION FROM AN SPHERICAL SOURCE IN THE KELLER-MIKSIS MODEL}

In order to predict the amplitude of the scattered plane wave of a bubble we assume the flow in the liquid to respect the Bernouilli equation (potential flow assumption)

$$
\frac{\partial \phi_{b}}{\partial t}+\frac{\partial \phi_{i}}{\partial t}+\frac{\partial \phi_{\infty}}{\partial t}+\frac{p_{l}}{\rho_{l_{0}}}+\frac{1}{2} u_{l}^{2}=\frac{p_{l_{0}}}{\rho_{l_{0}}},
$$

where $\phi_{b}$ represents the potential emitted by the bubble, $\phi_{i}$ is the interacting potential, and $\phi_{\infty}$ is the background potential that can be expressed as a function of the background excitation pressure as

$$
\frac{\partial \phi_{\infty}}{\partial t}=\frac{p_{l_{0}}-p_{\infty}}{\rho_{l_{0}}},
$$

where $p_{\infty}\left(P_{a}, t\right)$ corresponds to the background pressure used to excite the bubble. Evaluating the Bernouilli equation at the interface of the bubble we obtain

$$
\frac{\partial\left(\phi_{b}+\phi_{i}\right)}{\partial t}=\frac{p_{\infty}-p_{l, r=R}}{\rho_{l}}-\frac{1}{2} \dot{R}^{2} .
$$

The potential emitted by the bubble and the interacting potential behave as a wave. Based on the DNS results, we notice that the amplitude of the emitted wave decays with the distance until a given transition distance for which the amplitude of the emitted wave remains constant. If we assume that the transition distance from the spherical wave to the plane wave can be written as a function of the tube diameter through the use of a function $f\left(D / R_{0}\right)$, we can build a relation between the conditions obtained from KM model at the bubble interface and the intensity of the plane wave as:

$$
p_{l}-p_{\infty}=-\frac{1}{f\left(D / R_{0}\right)}\left[\frac{p_{\infty}\left(t^{\prime}\right)-p_{r=R}\left(t^{\prime}\right)}{\rho_{l_{0}}}-\frac{1}{2} R \dot{\left(t^{\prime}\right)^{2}}\right],
$$

where $t^{\prime}=t-x_{s} / c_{l}$. We obtain $f\left(D / R_{0}\right)$ by fitting the DNS

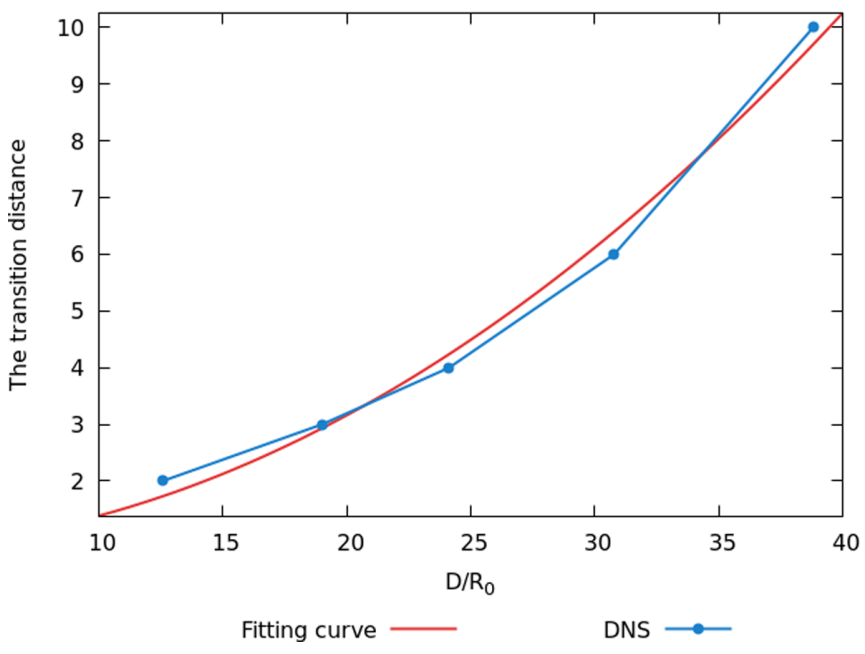

FIG. 14. Transition distance from a spherical to a plane wave as a function of $D / R_{0}$ obtained by DNS and fitted curve $f\left(D / R_{0}\right)$.

results shown in Fig. 14, where the transition distance is chosen as the first sample distance point of which the pressure attenuation deviates from the spherical attenuation. Following this procedure, we readily obtain $f\left(D / R_{0}\right)=0.005\left(\frac{D}{R_{0}}\right)^{2}+$ 0.8 .

\section{APPENDIX B: INFLUENCE OF RESONANT FREQUENCY SHIFT IN THE OSCILLATION AMPLITUDE}

Let us consider the dimensionless linearized RayleighPlesset equation written as

$$
\frac{\Delta R_{0}}{R_{0}}=\frac{-1}{\omega_{N}^{2}-\omega^{2}+2 \delta_{b} i \omega} \frac{\Delta p_{\infty}}{\rho_{l_{0}} R_{0}^{2}} \approx \frac{1}{\omega^{2}-\omega_{N}^{2}} \Delta p_{\infty},
$$

where $\Delta R_{0}$ is the oscillation amplitude in a free field and $\delta_{b}$ is the damping factor. If there is a change of the resonance frequency by bubble interactions such that $\omega_{N \text {,int }}=F \omega_{N}$, then when fixing $\omega=2 \omega_{N}$, the ratio between the oscillation amplitude with interactions $\Delta R$ and the oscillation amplitude in a free field becomes

$$
\frac{\Delta R}{\Delta R_{0}}=\frac{3}{4-F^{2}},
$$

meaning that the bubble oscillation is larger for a constant forcing amplitude when the frequency shifts to larger values $(F>1)$ and smaller in the opposite case $(F<1)$. Besides, by substituting $\omega=2 \omega_{N}$ into Eq. (B1), we can obtain the scaling factor

$$
\frac{\Delta R}{R_{0}}=\frac{P_{a}}{3 \rho_{l_{0}} \omega_{N}^{2} R_{0}^{2}},
$$

which in the dimensionless form chosen in this manuscript reads as $\Delta R=3 \omega_{N}^{2} \Delta p_{\infty}$.
[1] D. Lohse, Phys. Rev. Fluids 3, 110504 (2018).

[2] T. Shi, F. Forsberg, J. Raichlen, L. Needleman, and B. Goldberg, Ultrasound Med. Biol. 25, 275 (1999).
[3] P. Shankar, P. Krishna, and V. Newhouse, Ultrasound Med. Biol. 24, 395 (1998).

[4] B. P. Barber and S. J. Putterman, Nature (Lond.) 352, 318 (1991). 
[5] B. Gompf, R. Günther, G. Nick, R. Pecha, and W. Eisenmenger, Phys. Rev. Lett. 79, 1405 (1997).

[6] H. Lee and P. Choi, Ultrason. Sonochem. 6, 2037 (2014).

[7] W. Lauterborn and T. Kurz, Rep. Prog. Phys. 73, 1 (2010).

[8] A. Prosperetti, J. Acoust. Soc. Am. 56, 878 (1974).

[9] A. Prosperetti, J. Acoust. Soc. Am. 133, 3719 (2013).

[10] A. Katiyar and K. Sarkar, J. Acoust. Soc. Am. 130, 3137 (2011).

[11] K. Andersen and J. Jensen, J. Acoust. Soc. Am. 126, 3350 (2009).

[12] A. Katiyar, K. Sarkar, and F. Forsberg, J. Acoust. Soc. Am. 129 2325 (2011).

[13] T. Sun, N. Jia, D. Zhang, and D. Xu, J. Acoust. Soc. Am. 131, 4358 (2012).

[14] E. Biagi, L. Breschi, E. Vannacci, and L. Masotti, IEEE T. Ultrason. Ferr. 53, 2147 (2006).

[15] D. Zhang, X. Xi, Z. Zhang, X. Gong, G. Ghen, and J. Wu, Phys. Med. Biol. 54, 4257 (2009).

[16] V. Daeichin, T. Faez, G. Renaud, J. Bosch, A. V. der Steen, and N. de Jong, Phys. Med. Biol. 57, 3675 (2012).

[17] A. A. Doinikov, J. Fluid. Mech. 267, 1 (1994).

[18] M. Versluis, B. Schmitz, A. von der Heydt, and D. Lohse, Science 289, 2114 (2000).

[19] M. Ida, Phys. Rev. E 79, 016307 (2009).

[20] Y. Fan, H. Li, C. Xu, and T. Zhou, J. Acoust. Soc. Am. 145, 16 (2019).

[21] J. Liang, G. Han, L. Fengbin, and C. Darong, Ultrason. Sonochem. 34, 90 (2017).

[22] M. Guédra, C. Cornu, and C. Inserra, Ultrason. Sonochem. 38, 168 (2017).

[23] R. Mettin, I. Akhatov, U. Parlitz, C. D. Ohl, and W. Lauterborn, Phys. Rev. E 56, 2924 (1997).
[24] K. Yasui, Y. Iida, T. Tuziuti, T. Kozuka, and A. Towata, Phys. Rev. E 77, 016609 (2008).

[25] Y. An, Phys. Rev. E 85, 016305 (2012).

[26] Y. Fan, H. Li, J. Zhu, and W. Du, Ultrason. Sonochem. 1, 104790 (2019).

[27] Y. A. Ilinskii, M. F. Hamilton, and E. A. Zabolotskaya, J. Acoust. Soc. Am. 121, 786 (2007).

[28] D. Fuster, Flow, Turbul. Combust. 102, 497 (2019).

[29] D. Fuster, J. M. Conoir, and T. Colonius, Phys. Rev. E 90, 063010 (2014).

[30] D. Fuster and T. Colonius, J. Fluid Mech. 688, 352 (2011).

[31] V. Leroy, A. Strybulevych, M. Scanlon, and J. Page, Eur. Phys. J. E 29, 123 (2009).

[32] M. Miksis and L. Ting, J. Acoust. Soc. Am. 86, 2349 (1989).

[33] D. Fuster and S. Popinet, J. Comput. Phys. 347, 752 (2018).

[34] S. Popinet, J. Comput. Phys. 302, 336 (2015).

[35] J. B. Keller and M. Miksis, J. Acoust. Soc. Am. 68, 628 (1980)

[36] D. Fuster, C. Dopazo, and G. Hauke, J. Acoust. Soc. Am. 129, 122 (2011).

[37] S. Hilgenfeldt, D. Lohse, and M. Brenner, Phys. Fluids 8, 2808 (1996).

[38] Q. X. N. Amanda, A. Faraci, K. Christensen-Jeffries, J. L. Raymond, M. J. Monaghan, D. Fuster, F. Forsberg, R. J. Eckersley, and P. Lamata, IEEE T Ultrason. Ferr. 67, 557 (2019).

[39] A. A. Doinikov and P. Marmottant, J. Sound Vib. 420, 61 (2018).

[40] O. Lombard, C. Barrire, and V. Leroy, Euro. Phys. L. 112, 24002 (2015). 